\title{
Exponential Fitted Operator Method for Singularly Perturbed Convection-Diffusion Type Problems with Nonlocal Boundary Condition
}

\author{
Habtamu Garoma Debela \\ Department of Mathematics, College of Natural Sciences, Jimma University, Jimma, Ethiopia \\ Correspondence should be addressed to Habtamu Garoma Debela; habte200@gmail.com
}

Received 23 January 2021; Accepted 3 March 2021; Published 25 March 2021

Academic Editor: Lucas Jodar

Copyright (C) 2021 Habtamu Garoma Debela. This is an open access article distributed under the Creative Commons Attribution License, which permits unrestricted use, distribution, and reproduction in any medium, provided the original work is properly cited.

\begin{abstract}
This paper presents the study of singularly perturbed differential equations of convection-diffusion type with nonlocal boundary condition. The proposed numerical scheme is a combination of the classical finite difference method for the boundary conditions and exponential fitted operator method for the differential equations at the interior points. Maximum absolute errors and rates of convergence for different values of perturbation parameter and mesh sizes are tabulated for the numerical examples considered. The method is shown to be first-order accuracy independent of the perturbation parameter $\varepsilon$.
\end{abstract}

\section{Introduction}

Singularly perturbed differential equations are typically characterized by the presence of a small positive parameter $\varepsilon$ multiplying some or all of the highest order terms in differential equations. Such types of problems arise frequently in mathematical models of different areas of physics, chemistry, biology, engineering science, economics, and even sociology. The well-known examples are the heat transfer problem with large Peclet numbers, semiconductor theory, chemical reactor theory, reaction-diffusion process, theory of plates, optimal control, aerodynamics, seismology, oceanography, meteorology, and geophysics. Solutions of such equations usually possess thin boundary or interior layers where the solutions change very rapidly, while away from the layers, the solutions behave regularly and change slowly. More details about these problems can be found in [1-4] and also the literature cited there. Due to the presence of these boundary layers, the usual numerical treatment of singularly perturbed problems gives rise to computational difficulties. Standard numerical methods are not appropriate for practical applications when the perturbation parameter $\varepsilon$ is sufficiently small. Therefore, it is necessary to develop suitable numerical methods that are uniformly convergent with respect to $\varepsilon$ : To solve these problems, there are generally two types of approaches, such as fitted operator methods that reflect the nature of the solution in the boundary layers and fitted mesh methods which use layer-adapted meshes. In recent years, many authors have worked for solving singularly perturbed problems with one or two boundary layers using uniformly convergent numerical methods [5-11]. Boundary value problems including nonlocal conditions which connect the values of the unknown solution at the boundary with values in the interior are known as nonlocal boundary value problems. The study of this kind of problems was initiated by Il'in and Moiseev in [12,13], motivated by the work of Bitsadze and Samarskii on nonlocal linear elliptic boundary value problems [14]. These problems have been used to represent mathematical models of a large number of phenomena, such as problems of semiconductors in electronics, the vibrations of a guy wire of a uniform cross-section, heat transfer problems, problems of hydromechanics, catalytic processes in chemistry and biology, the diffusiondrift model of semiconducting devices, and some other physical phenomena [15-17]. The existence and uniqueness of the solutions of nonlocal boundary value problems have been 
studied by many authors $[18,19]$. Some approaches for the numerical solution of singularly perturbed nonlocal boundary value problems have been proposed in [20-28]. Uniformly convergent numerical methods of order second and high for solving different singularly perturbed problems have been studied in [29-34]. To the best of our knowledge, the problem under consideration has not been done using the fitted operator method. Motivated by papers [35, 36], we develop a uniformly convergent numerical method for solving singularly perturbed problem under consideration.

\section{Statement of the Problem}

Consider the following singularly perturbed problem with nonlocal condition of the form

$$
L y(x) \equiv \varepsilon y^{\prime \prime}(x)+a(x) y^{\prime}(x)=f(x), \quad 0<x<l,
$$

with the given conditions

$$
\begin{gathered}
y^{\prime}(0)=\frac{A}{\varepsilon}, \\
y(0)+\gamma y\left(l_{1}\right)=B y(l)+d,
\end{gathered}
$$

where $0<\varepsilon \ll 1$ is a small positive parameter, $A, B, \gamma$, and $d$ are given constants, $l_{1}$ and $l$ are given real numbers, and $\Omega$ $=(0,1)$ and $\bar{\Omega}=[0, l]$. We assume that $a(x) \geq a>0$ and $f(x$ ) are sufficiently smooth functions on $\bar{\Omega}$. Under these assumptions, singularly perturbed nonlocal Equations (1)-(3) possess a unique solution indicating a boundary layer of exponential type at $x=0$.

\section{Properties of Continuous Solution}

The following lemmas [13] are necessary for the existence and uniqueness of the solution and for the problem to be well posed.

Lemma 1 (continuous minimum principle). Assume that $v($ $x) \in C^{2}(\bar{\Omega})$ is any function satisfying $v(0) \geq 0, v(l) \geq 0$, and $L v(x) \leq 0, \forall x \in \Omega=(0, l)$. Then, $v(x) \geq 0, \forall x \in \Omega=[0, l]$.

Proof. Let $x^{*}$ be such that $v\left(x^{*}\right)=\min _{x \in[0, l]} v(x)$ and assume that $v\left(x^{*}\right)<0$. Clearly, $x^{*} \notin\{0, l\}$; therefore, $v^{\prime}\left(x^{*}\right)=0$ and $v^{\prime \prime}\left(x^{*}\right) \geq 0$. Moreover, $\operatorname{Lv}\left(x^{*}\right)=\varepsilon v^{\prime \prime}\left(x^{*}\right)+a\left(x^{*}\right) v^{\prime}\left(x^{*}\right) \geq 0$, which is a contradiction. It follows that $v\left(x^{*}\right)>0$, and thus, $v(x) \geq 0, \forall x \in[0, l]$.

The uniqueness of the solution is implied by this minimum principle. Its existence follows trivially (as for linear problems, the uniqueness of the solution implies its existence). This principle is now applied to prove that the solution of Equations (1)-(3) is bounded. The following lemma shows the bound for the derivatives of the solution.

Lemma 2. Let $y_{\varepsilon}$ be the solution of $\left(P_{\varepsilon}\right)$. Then, for $k=0,1,2,3$,

$$
\left|y_{\varepsilon}^{(k)}(x)\right| \leq C\left(1+\varepsilon^{-k} \exp \left(\frac{-a}{\varepsilon} x\right)\right), \quad \forall x \in[0, l]
$$

Proof. The homogeneous differential equation of Equation (1) is

$$
\varepsilon y^{\prime \prime}(x)+a(x) y^{\prime}(x)=0 \text {. }
$$

The characteristic equation of Equation (5) is

$$
\varepsilon m^{2}+a m=0 \Rightarrow m=0
$$

or

$$
m=\frac{-a}{\varepsilon}
$$

The asymptotic solution of Equation (5) is given by

$$
u(x)=A+B \exp \left(\frac{-a}{\varepsilon} x\right)
$$

where $A$ and $B$ are arbitrary constant.

To get the $k^{\text {th }}$ derivative of the asymptotic solution of the homogeneous part of Equation (5),

$$
\begin{aligned}
& u^{\prime}(x)=C \varepsilon^{-1} \exp \left(\frac{-a}{\varepsilon} x\right), \\
& u^{\prime \prime}(x)=C \varepsilon^{-2} \exp \left(\frac{-a}{\varepsilon} x\right), \\
& u^{\prime \prime}(x)=C \varepsilon^{-3} \exp \left(\frac{-a}{\varepsilon} x\right) .
\end{aligned}
$$

In general, for $k=1,2,3$,

$$
u^{(k)}(x)=C \varepsilon^{-k} \exp \left(\frac{-a}{\varepsilon} x\right)
$$

The reduced problem obtained from Equation (1) takes $a(x) v_{0}{ }^{\prime}(x)=f(x)$, where $v_{0}(0)=y_{0}$ and has the solution

$$
\begin{aligned}
v_{0}(x) & =y_{0}+\int_{0}^{x} \frac{f(t)}{a(t)} d t\left|v_{0}(x)\right| \leq\left|y_{0}\right|+\int_{0}^{x}\left|\frac{f(t)}{a(t)}\right| d t \leq C+\left|\frac{f(\zeta)}{a(\zeta)}\right| \int_{0}^{x} d t \\
& \leq C+\left|\frac{f(\zeta)}{a(\zeta)}\right| x, x \in(0, l), \leq C ;
\end{aligned}
$$

from the assumptions on $a$ and $f$, it is clear that for $k=$ $0,1,2,3$,

$$
\left|v_{0}^{(k)}(x)\right| \leq C, \quad \forall x \in[0,1]
$$

So, from the relation $y_{\varepsilon}=v_{0}+u$, we have $y_{\varepsilon}^{(k)}=v_{0}^{(k)}+u^{(k)}$, and from the relation of triangular inequality,

$$
\left|y_{\varepsilon}^{(k)}\right| \leq\left|v_{0}^{(k)}\right|+\left|u^{(k)}\right| \leq C+C \varepsilon^{-k} \exp \left(\frac{-a}{\varepsilon} x\right) \leq C\left(1+\varepsilon^{-k} \exp \left(\frac{-a}{\varepsilon} x\right)\right) .
$$

Therefore, it is well accepted that the solution of Equation 
(1) has a boundary layer near $x=0$ and its derivatives satisfy

$$
\left|y_{\varepsilon}^{(k)}(x)\right| \leq C\left(1+\varepsilon^{-k} \exp \left(\frac{-a}{\varepsilon} x\right)\right), \quad \forall x \in[0, l] .
$$

\section{Formulation of the Method}

Consider the homogeneous differential equation with constant coefficient $\varepsilon y^{\prime \prime}(x)+a y^{\prime}(x)=0$ whose solution is given by

$$
y(x)=A+B \exp \left(\frac{-a}{\varepsilon} x\right)
$$

where $A$ and $B$ are constants which will be determined depending on the given conditions. Now, dividing the interval $[0, l]$ into $N$ equal parts with constant mesh length $h=l / N$ , we obtain $x_{i}=x_{0}+i h$, for $i=1,2, \cdots, N$, where $x_{0}=0, x_{N}=l$.

To demonstrate the procedure, we consider Equation (1), at discrete nodes $x_{i}$

$$
\varepsilon y_{i}^{\prime \prime}(x)+a\left(x_{i}\right) y_{i}^{\prime}(x)=f\left(x_{i}\right) .
$$

Approximating Equation (16) by central difference approximations, we obtain

$$
\varepsilon \frac{y_{i-1}-2 y_{i}+y_{i+1}}{h^{2}}+a_{i} \frac{y_{i+1}-y_{i-1}}{2 h}=f_{i} .
$$

Under the assumption that $f_{i}$ is bounded, introducing the fitting parameter $\sigma$ onto the higher order difference approximation of Equation (17), multiply both sides by $h$, and evaluating its limit gives

$$
\sigma=\frac{\rho a \lim _{h \rightarrow 0}\left(y_{i+1}-y_{i-1}\right)}{2 \lim _{h \rightarrow 0}\left(y_{i+1}-2 y_{i}+y_{i-1}\right)},
$$

where $\rho=h / \varepsilon$.

Evaluating Equation (15) at each nodal points $x_{i}$, we obtain

$$
\left\{\begin{array}{l}
\lim _{h \rightarrow 0} y_{i}=A\left(1-\exp \left(-\frac{1}{\varepsilon}\right) \exp (-i \rho)\right)-B \exp \left(-\frac{1}{\varepsilon}\right)(1-\exp (-i \rho)), \\
\lim _{h \rightarrow 0} y_{i+1}=A\left(1-\exp \left(-\frac{1}{\varepsilon}\right) \exp (-i \rho)\right)-B \exp \left(-\frac{1}{\varepsilon}\right)(1-\exp (-i \rho)), \\
\lim _{h \rightarrow 0} y_{i-1}=A\left(1-\exp \left(-\frac{1}{\varepsilon}\right) \exp (-i \rho)\right)-B \exp \left(-\frac{1}{\varepsilon}\right)(1-\exp (-i \rho)),
\end{array}\right.
$$

$$
\sigma=\frac{\rho a \lim _{h \rightarrow 0}\left(y_{i+1}-y_{i-1}\right)}{2 \lim _{h \rightarrow 0}\left(y_{i+1}-2 y_{i}+y_{i-1}\right)}=\frac{a \rho}{2} \operatorname{coth}\left(\frac{a \rho}{2}\right) .
$$

Hence, from Equations (17) and (20), we get

$$
\frac{\varepsilon \sigma}{h^{2}}\left(y_{i-1}-2 y_{i}+y_{i+1}\right)+\frac{a_{i}}{2 h}\left(y_{i+1}-y_{i-1}\right)=f_{i} .
$$

This can be rewritten as three-term recurrence relation:

$$
E_{i} y_{i-1}+F_{i} y_{i}+G_{i} y_{i+1}=H_{i}, \quad i=1,2, \cdots, N-1,
$$

where

$$
\left\{\begin{array}{l}
E_{i}=\frac{\varepsilon \sigma}{h^{2}}-\frac{a_{i}}{2 h}, \\
F_{i}=\frac{-2 \varepsilon \sigma}{h^{2}}, \\
G_{i}=\frac{\varepsilon \sigma}{h^{2}}+\frac{a_{i}}{2 h}, \\
H_{i}=f_{i} .
\end{array}\right.
$$

Since the problem involves nonlocal boundary conditions, we considered the following cases, to obtain two equations at each end conditions.

For $i=0$, Equation (22) becomes

$$
E_{0} Y_{-1}+F_{0} Y_{0}+G_{0} Y_{1}=H_{0}
$$

Here, in Equation (24), the term $Y_{-1}$ is out of the domain, so that using Equation (2), we have

$$
Y^{\prime}(0)=\frac{\mu_{0}}{\varepsilon}=\frac{Y_{1}-Y_{-1}}{2 h} \Rightarrow Y_{-1}=Y_{1}-2 h Y^{\prime}(0) \text {. }
$$

Putting Equation (25) into Equation (24) gives

$$
E_{0} Y_{0}+\left(E_{0}+G_{0}\right) Y_{1}=H_{0}+2 h E_{0} Y^{\prime}(0)
$$

For $i=N$, Equation (22) becomes

$$
E_{N} Y_{N-1}+F_{N} Y_{N}+G_{N} Y_{N+1}=H_{N}
$$

Here, in Equation (27), the term $Y_{N+1}$ is out of the domain, so that using Equation (3), we have

$$
y_{N+1}=\frac{y_{0}}{B}+\frac{\gamma Y_{l_{1}}}{B}-\frac{d}{B} .
$$

Putting Equation (28) into Equation (27) gives

$$
\frac{G_{N}}{B} Y_{0}+\frac{G_{N} \gamma}{B} Y_{l_{1}}=H_{N}+\frac{G_{N} d}{B} .
$$

Therefore, Equation (1) with the given boundary conditions in Equations (2) and (3) can be solved using the schemes in Equations (22), (26), and (29) which gives $N \times$ $N$ system of algebraic equations.

\section{Uniform Convergence Analysis}

In this section, we need to show the discrete scheme in Equation (22) and satisfy the discrete minimum principle, uniform stability estimates, and uniform convergence. 
Lemma 3 (discrete minimum principle). Let $Y_{i}$ be any mesh function that satisfies $Y_{0} \geq 0, Y_{N} \geq 0$, and $L_{\varepsilon}^{N} Y_{i} \leq 0, i=1,2$, $3, \cdots, N-1$, then $Y_{i} \geq 0$, for $i=0,1,2, \cdots, N$.

Proof. The proof is by contradiction. Let $j$ be such that $Y_{j}=$ $\min _{i} Y_{i}$ and suppose that $Y_{j} \leq 0$. Clearly, $j \notin\{0, N\} . Y_{j+1}-$ $Y_{j} \geq 0$ and $Y_{j}-Y_{j-1} \leq 0$.

Therefore,

$$
\begin{aligned}
L_{\varepsilon}^{N} Y_{j} & =\varepsilon\left(\frac{Y_{j+1}-2 Y_{j}+Y_{j-1}}{\Psi_{i}^{2}}\right)+a_{j}\left(\frac{Y_{j+1}-Y_{j}}{h}\right) \\
& =\frac{\varepsilon}{\Psi_{i}^{2}}\left(Y_{j+1}-2 Y_{j}+Y_{j-1}\right)+\frac{a_{j}}{h}\left(Y_{j+1}-Y_{j}\right) \\
& =\frac{\varepsilon}{\Psi_{i}^{2}}\left(\left(Y_{j+1}-Y_{j}\right)-\left(Y_{j}-Y_{j-1}\right)\right)+\frac{a_{j}}{h}\left(Y_{j+1}-Y_{j}\right) \geq 0,
\end{aligned}
$$

where the strict inequality holds if $Y_{j+1}-Y_{j}>0$. This is a contradiction, and therefore, $Y_{j} \geq 0$. Since $j$ is arbitrary, we have $Y_{i} \geq 0$, for $i=0,1,2, \cdots, N$.

From the discrete minimum principle, we obtain an $\varepsilon-$ uniform stability property for the operator $L_{\varepsilon}^{N}$.

Lemma 4 (uniform stability estimate). If $\phi_{j}$ is any mesh function such that

$$
\phi_{0}=\phi_{N}=0, \text { then }\left|\phi_{j}\right| \leq \frac{1}{a} \max _{1 \leq i \leq N-1}\left|L_{\varepsilon}^{N} \phi_{i}\right|, j=0,1,2, \cdots, N .
$$

Proof. We introduce two mesh functions $\psi_{j}^{+}, \psi_{j}^{-}$defined by

$$
\psi_{j}^{ \pm}=\left(\frac{1}{a} \max _{1 \leq i \leq N-1}\left|L_{\varepsilon}^{N} \phi_{i}\right|\right) \pm \phi_{j}
$$

It follows that

$$
\begin{aligned}
\psi^{ \pm}(0) & =\left(\frac{1}{a} \max _{1 \leq i \leq N-1}\left|L_{\varepsilon}^{N} \phi_{i}\right|\right) \pm \phi_{0}=\frac{1}{a} \max _{1 \leq i \leq N-1}\left|\varepsilon \delta^{2} \phi_{i}+a_{i} D^{0} \phi_{i}\right| \pm \phi_{0} \\
& =\frac{1}{a} \max _{1 \leq i \leq N-1}\left|\varepsilon \delta^{2} \phi_{i}+a_{i} D^{0} \phi_{i}\right| \geq 0, \\
\psi^{ \pm}(N) & =\left(\frac{1}{a} \max _{1 \leq i \leq N-1}\left|L_{\varepsilon}^{N} \phi_{i}\right|\right) \pm \phi_{N}=\frac{1}{a} \max _{1 \leq i \leq N-1}\left|\varepsilon \delta^{2} \phi_{i}+a_{i} D^{0} \phi_{i}\right| \pm \phi_{N} \\
& =\frac{1}{a} \max _{1 \leq i \leq N-1}\left|\varepsilon \delta^{2} \phi_{i}+a_{i} D^{0} \phi_{i}\right| \geq 0,
\end{aligned}
$$

for all $j=1,2, \cdots, N-1$,

$$
L_{\varepsilon}^{N} \psi_{j}^{ \pm}=\left(\frac{1}{a} \max _{1 \leq i \leq N-1}\left|L_{\varepsilon}^{N} \phi_{i}\right|\right) \pm L_{\varepsilon}^{N} \phi_{j} \leq 0
$$

From a discrete minimum principle, if $\psi_{0} \geq 0, \psi_{N} \geq 0$, and $L_{\varepsilon}^{N} \psi_{j} \leq 0, \forall 0<j<N$, then $\psi_{j}^{ \pm} \geq 0,0 \leq j \leq N$.
We provide above that the discrete operator $L_{\varepsilon}^{N}$ satisfy the minimum principle. Next, we analyze the uniform convergence analysis.

Theorem 5. Let $y\left(x_{i}\right)$ and $Y_{i}$ be, respectively, the exact solution of Equations (1)-(3) and numerical solutions of Equation (22). Then, for sufficiently large $N$, the following parameter uniform error estimate holds:

$$
\left|L^{N}\left(y_{i}-Y_{i}\right)\right| \leq C h\left(1+\sup _{x \in(0,1)}\left(\frac{\exp \left(-a x_{i} / \varepsilon\right)}{\varepsilon^{3}}\right)\right) .
$$

Proof. Let us consider the local truncation error defined as

$$
\begin{aligned}
L^{N}\left(y\left(x_{i}\right)-Y_{i}\right)= & \varepsilon \sigma(\rho)\left(y^{\prime \prime}\left(x_{i}\right)-D^{+} D^{-} y\left(x_{i}\right)\right)+a\left(x_{i}\right)\left(y^{\prime}\left(x_{i}\right)-D^{0} y\left(x_{i}\right)\right) \\
= & \varepsilon\left[\frac{\rho a(0)}{2} \operatorname{coth}\left(\frac{\rho a(0)}{2}\right)-1\right] D^{+} D^{-} y\left(x_{i}\right) \\
& +\varepsilon\left(y^{\prime \prime}\left(x_{i}\right)-D^{+} D^{-} y\left(x_{i}\right)\right)+a\left(x_{i}\right)\left(y^{\prime}\left(x_{i}\right)-D^{0} y\left(x_{i}\right)\right),
\end{aligned}
$$

where $\sigma(\rho)=a(0)(\rho / 2) \operatorname{coth}(a(0) \rho / 2)$ and $\rho=N^{-1} / \varepsilon$.

Now, for $z>0, C_{1}$ and $C_{2}$ are constants, and we have $\mid z$ coth $(z)-1 \mid \leq C_{1} z^{2}, z \leq 1$. Similarly, for $z \rightarrow \infty$, since $\lim _{z \rightarrow \infty}$ $\operatorname{coth}(z)=1,|z \operatorname{coth}(z)-1| \leq C_{1} z$ is given.

In general, for all $z>0$, as [11], we write

$$
C_{1} \frac{z^{2}}{z+1} \leq z \operatorname{coth}(z)-1 \leq C_{2} \frac{z^{2}}{z+1}
$$

implying that

$$
\varepsilon\left[a(0) \frac{\rho}{2} \operatorname{coth}\left(a(0) \frac{\rho}{2}\right)-1\right] \leq \varepsilon\left(\frac{\left(N^{-1} / \varepsilon\right)^{2}}{\left(N^{-1} / \varepsilon\right)+1}\right)=\frac{N^{-2}}{N^{-1}+\varepsilon}
$$

Using Taylor series expansion, the bound for $y\left(x_{i-1}\right)$ and $y\left(x_{i+1}\right)$ at $x_{i}$ as

$$
\left\{\begin{array}{l}
y\left(x_{i-1}\right)=y\left(x_{i}\right)-h y^{\prime}\left(x_{i}\right)+\frac{h^{2}}{2 !} y^{\prime \prime}\left(x_{i}\right)-\frac{h^{3}}{3 !} y^{(3)}\left(x_{i}\right)+\frac{h^{4}}{4 !} y^{(4)}\left(x_{i}\right)+O\left(h^{5}\right), \\
y\left(x_{i+1}\right)=y\left(x_{i}\right)+h y^{\prime}\left(x_{i}\right)+\frac{h^{2}}{2 !} y^{\prime \prime}\left(x_{i}\right)+\frac{h^{3}}{3 !} y^{(3)}\left(x_{i}\right)+\frac{h^{4}}{4 !} y^{(4)}\left(x_{i}\right)+O\left(h^{5}\right) .
\end{array}\right.
$$

We obtain the bound for

$$
\left\{\begin{array}{l}
\left|D^{+} D^{-} y\left(x_{i}\right)\right| \leq C\left|y^{\prime \prime}\left(x_{i}\right)\right| \\
\left|y^{\prime \prime}\left(x_{i}\right)-D^{+} D^{-} y\left(x_{i}\right)\right| \leq C N^{-2}\left|y^{(4)}\left(x_{i}\right)\right|
\end{array}\right.
$$


TABLe 1: Maximum pointwise absolute errors, computed $\varepsilon$-uniform errors, and convergence rates for Example 8.

\begin{tabular}{lccccc}
\hline$\varepsilon$ & $N=16$ & $N=32$ & $N=64$ & $N=128$ & \multicolumn{1}{c}{$N=256$} \\
\hline $10^{-4}$ & $4.5340 e-03$ & $2.2114 e-03$ & $1.0919 e-04$ & $5.4256 e-04$ & $2.7046 e-04$ \\
$10^{-8}$ & $4.5342 e-03$ & $2.2115 e-03$ & $1.0920 e-03$ & $5.4258 e-04$ & $2.7044 e-04$ \\
$10^{-12}$ & $4.5342 e-03$ & $2.2115 e-03$ & $1.0920 e-03$ & $5.4258 e-04$ & $2.7044 e-04$ \\
$10^{-16}$ & $4.5342 e-03$ & $2.2115 e-03$ & $1.0920 e-03$ & $5.4258 e-04$ & $2.7044 e-04$ \\
$10^{-20}$ & $4.5342 e-03$ & $2.2115 e-03$ & $1.0920 e-03$ & $5.4258 e-04$ & $2.7044 e-04$ \\
$E^{N}$ & $4.5342 e-03$ & $2.2115 e-03$ & $1.0920 e-03$ & $5.4258 e-04$ & $2.7044 e-04$ \\
$R^{N}$ & 1.0358 & 1.0181 & 1.0091 & 1.0045 \\
\hline
\end{tabular}

TABle 2: Comparison of $\varepsilon$-uniform maximum absolute errors and $\varepsilon$-uniform rate of convergence for Example 8.

\begin{tabular}{lcccc}
\hline$\varepsilon$ & $N=16$ & $N=32$ & $N=64$ & $N=128$ \\
\hline Present method & & & & $N=256$ \\
$E^{N}$ & $4.5342 e-03$ & $2.2115 e-03$ & $1.0920 e-03$ & $5.4258 e-03$ \\
$R^{N}$ & 1.0358 & 1.0181 & 1.0091 & 1.0045 \\
Method in [36] & & & 0.0012892 \\
$E^{N}$ & 0.0104076 & 0.0051770 & 0.0025818 & 1.00 \\
$R^{N}$ & 1.01 & 1.00 & 1.00 & 0.000644 \\
\hline
\end{tabular}

TABle 3: Maximum pointwise absolute errors, computed $\mathcal{\varepsilon}$-uniform errors, and convergence rates for Example 9.

\begin{tabular}{lccccc}
\hline$\varepsilon$ & $N=16$ & $N=32$ & $N=64$ & $N=128$ & \multicolumn{1}{c}{$N=256$} \\
\hline $10^{-4}$ & $4.1080 e-03$ & $2.0018 e-03$ & $9.8802 e-04$ & $4.9081 e-04$ & $2.4426 e-04$ \\
$10^{-8}$ & $4.1082 e-03$ & $2.0019 e-03$ & $9.8807 e-04$ & $4.9083 e-04$ & $2.4463 e-04$ \\
$10^{-12}$ & $4.1082 e-03$ & $2.0019 e-03$ & $9.8807 e-04$ & $4.9083 e-04$ & $2.4462 e-04$ \\
$10^{-16}$ & $4.1082 e-03$ & $2.0019 e-03$ & $9.8807 e-04$ & $4.9083 e-04$ & $2.4462 e-04$ \\
$10^{-20}$ & $4.1082 e-03$ & $2.0019 e-03$ & $9.8807 e-04$ & $4.9083 e-04$ & $2.4462 e-04$ \\
$E^{N}$ & $4.1082 e-03$ & $2.0019 e-03$ & $9.8807 e-04$ & $4.9083 e-04$ & $2.4462 e-04$ \\
$R^{N}$ & 1.0371 & 1.0187 & 1.0094 & 1.0047 \\
\hline
\end{tabular}

TABLE 4: Comparison of $\varepsilon$-uniform maximum absolute errors and $\varepsilon$-uniform rate of convergence for Example 9.

\begin{tabular}{lcccc}
\hline$\varepsilon$ & $N=16$ & $N=32$ & $N=64$ & $N=128$ \\
\hline $\begin{array}{l}\text { Present method } \\
E^{N}\end{array}$ & $4.1082 e-03$ & $2.0019 e-03$ & $9.8807 e-04$ & $4.9083 e-04$ \\
$R^{N}$ & 1.0371 & 1.0187 & 1.0094 & 1.0047 \\
Method in [36] & & & & $2.4462 e-04$ \\
$E^{N}$ & 0.0116499 & 0.0057949 & 0.0028900 & 0.0014431 \\
$R^{N}$ & 1.01 & 1.00 & 1.00 & 1.00 \\
\hline
\end{tabular}




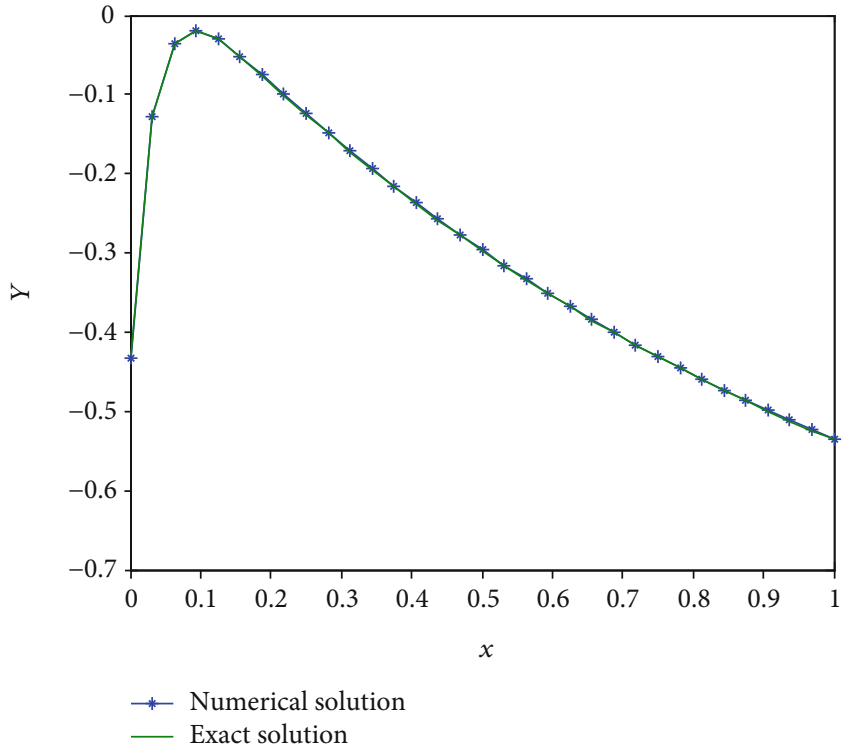

(a)

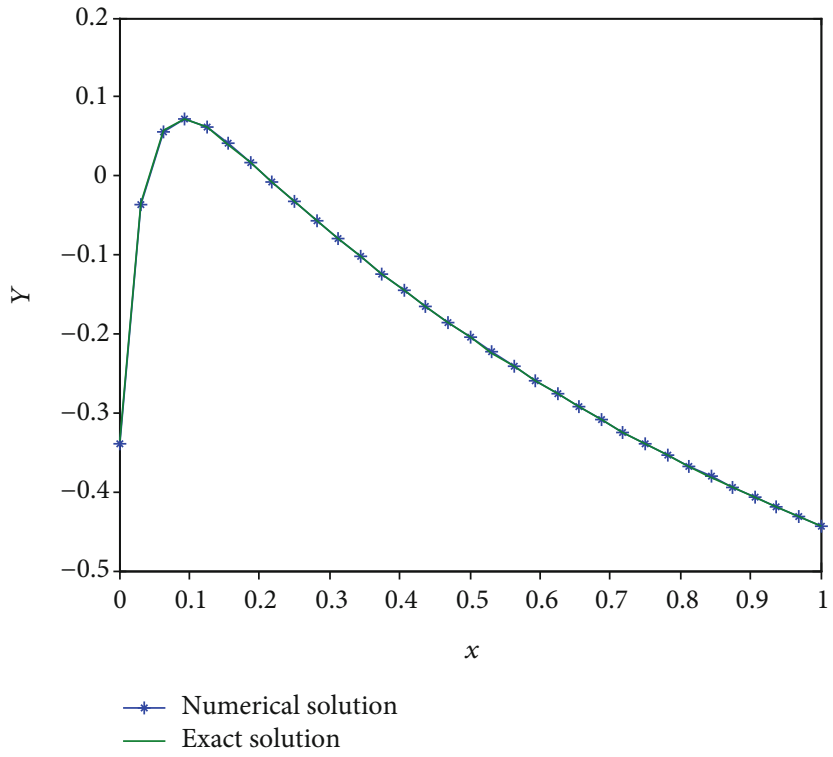

(b)

Figure 1: The behavior of the numerical solution at $\varepsilon=2^{-4}$ and $N=32$ of Examples 8 and 9, respectively.

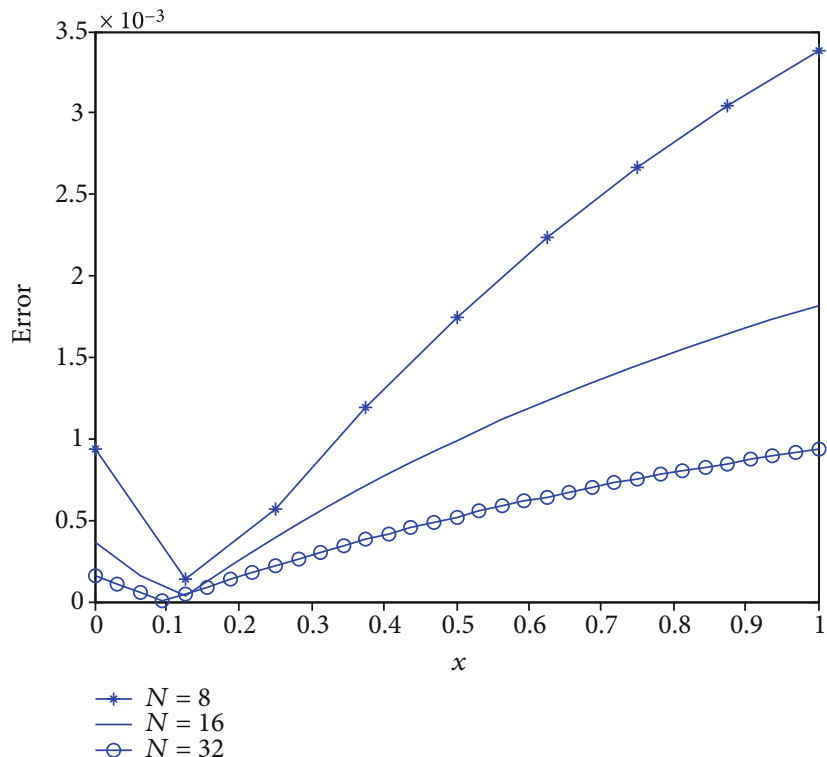

(a)

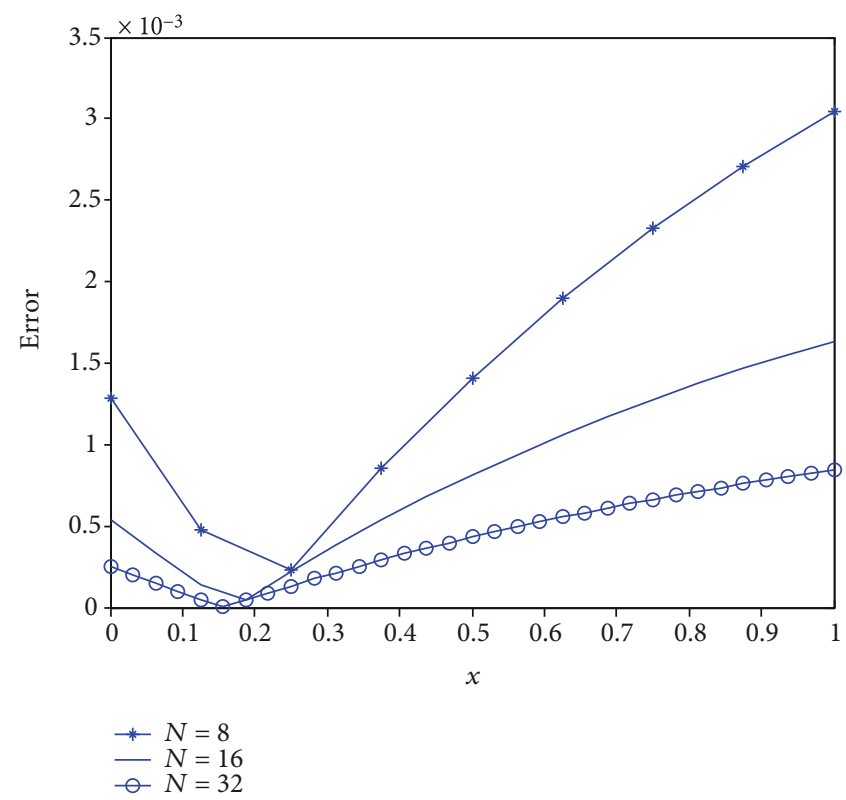

(b)

Figure 2: Pointwise absolute error of Examples 8 and 9 at $\varepsilon=10^{-20}$ with different mesh points $N$, respectively.

Similarly, for the first derivative term,

$$
\left|y^{\prime}\left(x_{i}\right)-D^{0} y\left(x_{i}\right)\right| \leq C N^{-2}\left|y^{(3)}\left(x_{i}\right)\right|
$$

where $\left|y^{(k)}\left(x_{i}\right)\right|=\sup _{x_{i} \in\left(x_{0}, x_{N}\right)}\left|y^{(k)}\left(x_{i}\right)\right|, k=2,3,4$.
Using the bounds in Equations (40) and (41), we obtain

$$
\begin{aligned}
\left|L^{N}\left(y\left(x_{i}\right)-Y_{i}\right)\right| & \leq \frac{C N^{-2}}{N^{-1}+\varepsilon}\left|y^{\prime \prime}\left(x_{i}\right)\right|+\varepsilon C N^{-2}\left|y^{(4)}\left(x_{i}\right)\right|+C N^{-2}\left|y^{(3)}\left(x_{i}\right)\right| \\
& \leq \frac{C N^{-2}}{N^{-1}+\varepsilon}\left|y^{\prime \prime}\left(x_{i}\right)\right|+C N^{-2}\left[\varepsilon\left|y^{(4)}\left(x_{i}\right)\right|+\left|y^{(3)}\left(x_{i}\right)\right|\right] .
\end{aligned}
$$




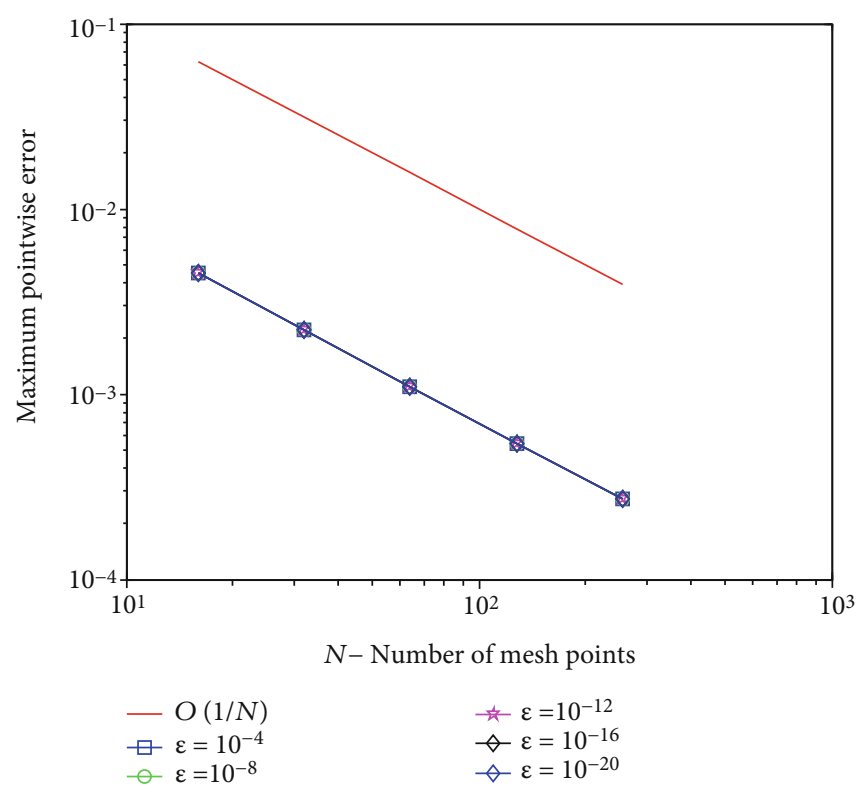

(a)

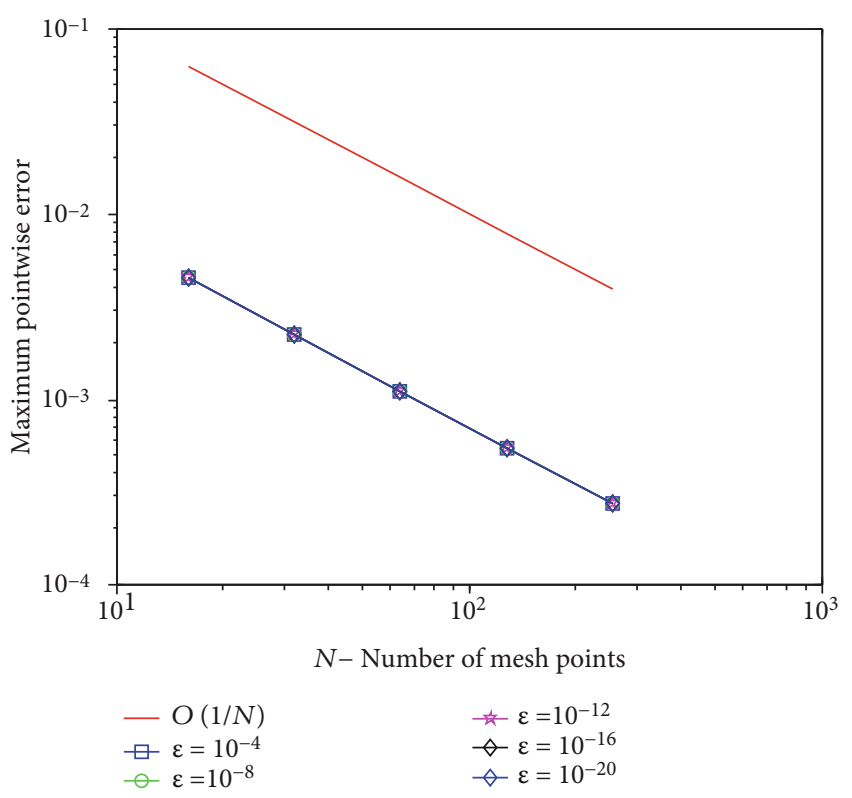

(b)

FIGURE 3: $\varepsilon$-uniform convergence with exponential fitted operator method in log-log scale for Examples 8 and 9, respectively.

Now, using the bounds for the derivatives of the solution in Lemma (2), we have

$$
\begin{aligned}
\left|L^{N}\left(y\left(x_{i}\right)-Y_{i}\right)\right| \leq & \frac{C N^{-2}}{N^{-1}+\varepsilon}\left(1+\varepsilon^{-2}\left(\exp \left(\frac{-c x_{i}}{\varepsilon}\right)\right)\right)+C N^{-2} \\
& \cdot\left[\varepsilon\left(1+\varepsilon^{-4}\left(\exp \left(\frac{-c x_{i}}{\varepsilon}\right)\right)\right)+\left(1+\varepsilon^{-3}\left(\exp \left(\frac{-c x_{i}}{\varepsilon}\right)\right)\right)\right] \\
\leq & \frac{C N^{-2}}{N^{-1}+\varepsilon}\left(1+\varepsilon^{-2}\left(\exp \left(\frac{-c x_{i}}{\varepsilon}\right)\right)\right)+C N^{-2} \\
& \cdot\left[\left(\varepsilon+\varepsilon^{-3}\left(\exp \left(\frac{-c x_{i}}{\varepsilon}\right)\right)\right)+\left(1+\varepsilon^{-3}\left(\exp \left(\frac{-c x_{i}}{\varepsilon}\right)\right)\right)\right],
\end{aligned}
$$

which simplifies to

$$
\left|L^{N}\left(y\left(x_{i}\right)-Y_{i}\right)\right| \leq \frac{C N^{-2}}{N^{-1}+\varepsilon}\left(1+\varepsilon^{-3}\left(\exp \left(\frac{-c_{1} x_{i}}{\varepsilon}\right)\right)\right)
$$

since $\varepsilon^{-3} \geq \varepsilon^{-2}$

Lemma 6. For a fixed mesh and for $\varepsilon \rightarrow 0$, it holds

$$
\begin{gathered}
\lim _{\varepsilon \rightarrow 0} \max _{1 \leq i \leq N-1} \frac{\exp \left(-a x_{i} / \varepsilon\right)}{\varepsilon^{m}}=0, \quad m=1,2,3, \cdots, \\
\lim _{\varepsilon \rightarrow 0} \max _{1 \leq i \leq N-1} \frac{\exp \left(-a\left(1-x_{i}\right) / \varepsilon\right)}{\varepsilon^{m}}=0, \quad m=1,2,3, \cdots .
\end{gathered}
$$

Proof. Consider the partition $[0,1]:=\left\{0=x_{0}<x_{1}<\cdots<x_{N-1}\right.$
$\left.<x_{N}=1\right\}$ for the interior grid points, we have

$$
\begin{aligned}
\max _{1 \leq i \leq N-1} \frac{\exp \left(-a x_{i} / \varepsilon\right)}{\varepsilon^{m}} & \leq \frac{\exp \left(-a x_{1} / \varepsilon\right)}{\varepsilon^{m}}=\frac{\exp (-a h / \varepsilon)}{\varepsilon^{m}}, \\
\max _{1 \leq i \leq N-1} \frac{\exp \left(-a\left(1-x_{i}\right) / \varepsilon\right)}{\varepsilon^{m}} & \leq \frac{\exp \left(-a\left(1-x_{N-1}\right) / \varepsilon\right)}{\varepsilon^{m}}=\frac{\exp (-a h / \varepsilon)}{\varepsilon^{m}}, \\
a s x_{1} & =1-x_{N-1}=h .
\end{aligned}
$$

The repeated application of L'Hospital's rule gives

$$
\lim _{\varepsilon \rightarrow 0} \frac{\exp (-a h / \varepsilon)}{\varepsilon^{m}}=\lim _{r=1 / \varepsilon \rightarrow \infty} \frac{r^{m}}{\exp (a h r)}=\lim _{r=1 / \varepsilon \rightarrow \infty} \frac{m !}{(a h)^{m} \exp (a h r)}=0 .
$$

This completes the proof.

Theorem 7. Let $y\left(x_{i}\right)$ and $Y_{i}$ be the exact solution of Equations (1)-(3) and numerical solutions of Equation (22), respectively. Then, the following error bound holds

$$
\sup _{0<\varepsilon<1}\left|\left(y\left(x_{i}\right)-Y_{i}\right)\right| \leq \frac{C N^{-2}}{N^{-1}+\varepsilon} \leq C N^{-1} .
$$

Proof. By substituting the results in Lemma 6 into Theorem 5 and applying the discrete maximum principle, we obtain the required bound.

For the case $\varepsilon>N^{-1}$, the scheme secures second-order convergence, and we expect to lose an order of convergence for $\varepsilon \leq N^{-1}$, and in fact, it turns out that the scheme is firstorder uniformly convergent. 


\section{Numerical Example and Results}

To validate the established theoretical results, we perform numerical experiments using the model problems of the form in Equations (1)-(3).

Example 8. Consider the model singularly perturbed boundary value problem:

$$
\varepsilon y^{\prime \prime}(x)+2 y^{\prime}(x)=(\varepsilon-2) e^{-x}, \quad 0<x<1,
$$

subject to the boundary conditions

$$
\begin{gathered}
y^{\prime}(0)=\frac{1}{\varepsilon}, \\
y(0)+\frac{1}{3} y\left(\frac{1}{4}\right)+y(1)=1 .
\end{gathered}
$$

Its exact solution is

$$
y(x)=d_{1}+d_{2} e^{-2 x / \varepsilon}+e^{-x},
$$

where $d_{1}=-3 / 7\left[e^{-1}+(1 / 3) e^{-1 / 4}+\left(1+e^{-2 x / \varepsilon}+(1 / 3) e^{-1 / 2 \varepsilon}\right) d_{2}\right.$ ], $d_{2}=-(1+\varepsilon) / 2$.

Example 9. Consider the model singularly perturbed boundary value problem:

$$
\varepsilon y^{\prime \prime}(x)+2 y^{\prime}(x)=(\varepsilon-2) e^{-x}, \quad 0<x<1,
$$

subject to the boundary conditions

$$
\begin{gathered}
y^{\prime}(0)=\frac{1}{\varepsilon}, \\
y(0)+\frac{2}{3} y\left(\frac{3}{4}\right)+y(1)=1 .
\end{gathered}
$$

Its exact solution is

$$
y(x)=d_{1}+d_{2} e^{-2 x / \varepsilon}+e^{-x},
$$

where $d_{1}=-3 / 8\left[e^{-1}+(2 / 3) e^{-3 / 4}+\left(1+e^{-2 x / \varepsilon}+(2 / 3) e^{-3 / 2 \varepsilon}\right) d_{2}\right.$ ] and $d_{2}=-(1+\varepsilon) / 2$.

We define the pointwise absolute errors $E_{\varepsilon}^{N}$ and the computed $\varepsilon$-uniform maximum pointwise error $E^{N}$ as follows:

$$
E_{\varepsilon}^{N}=\|Y-y\|, \quad E^{N}=\max _{\varepsilon} E_{\varepsilon}^{N}
$$

where $Y$ is the numerical approximation to $y$ for various values of $N$ and $\varepsilon$. We also define the computed $\varepsilon$-uniform convergence rate:

$$
R^{N}=\log _{2} \frac{E^{N}}{E^{2 N}} .
$$

\section{Discussion and Conclusion}

This study introduces a uniformly convergent numerical method based on the exponential fitted operator method for solving singularly perturbed boundary value problems with nonlocal boundary condition. The behavior of the continuous solution of the problem is studied and shows that it satisfies the continuous stability estimate, and the derivatives of the solution are also bounded. The numerical scheme is developed on uniform mesh. The nonlocal boundary condition is treated using the finite difference formula, and the results are compared accordingly. The stability of the developed scheme is established, and its uniform convergence is proved. To validate the applicability of the method, two model problems are considered for numerical experimentation for different values of the perturbation parameter and mesh points. The numerical results are tabulated in terms of maximum absolute errors, numerical rate of convergence, and uniform errors (see Tables 1-4) and compared with the results of the previously developed numerical methods existing in the literature (Tables 2 and 4). Further, the behavior of the numerical solutions is plotted in Figure 1; Figure 2 shows that as the mesh size decreases or as the number of nodal points increases, point wise absolute error decreases; and Figure 3 shows that our method is $\varepsilon$-uniformly convergent independent of the mesh size for $h \geq \varepsilon$, where the classical numerical methods fail in the log-log scale. Unlike other fitted operator finite difference methods constructed in standard ways, the method that we presented in this paper is fairly simple to construct. Moreover, the method is more accurate and gives a good result where existing numerical methods fail (that is for the values where the perturbation parameter, $\varepsilon$, is much less than the mesh size, $h$ ).

\section{Data Availability}

No data were used to support this study.

\section{Conflicts of Interest}

The authors declare that they have no conflicts of interest.

\section{Acknowledgments}

The author wish to express their thanks to Jimma University, College of Natural Sciences, for financial support, and the authors of the literature for the provided scientific aspects and idea for this work.

\section{References}

[1] J. Kevorkian and J. D. Cole, Multiple Scale and Singular Perturbation Methods, vol. 114 of Applied Mathematical Sciences, Springer, New York, 1996.

[2] A. H. Nayfeh, Perturbation Methods, Wiley, New York, 1985.

[3] R. E. O'Malley, Singular Perturbation Methods for Ordinary Differential Equations, Springer Verlag, New York, 1991.

[4] D. R. Smith, Singular Perturbation Theory: an Introduction with Applications, Cambridge University Press, Cambridge, 1985. 
[5] E. P. Doolan, J. J. H. Miller, and W. H. A. Schilders, Uniform Numerical Method for Problems with Initial and Boundary Layers, Boole Press, Dublin, 1980.

[6] P. Farrell, A. Hegarty, J. M. Miller, E. O'Riordan, and G. I. Shishkin, Robust Computational Techniques for Boundary Layers, Chapman Hall/CRC, New York, 2000.

[7] M. K. Kadalbajoo and V. Gupta, "A brief survey on numerical methods for solving singularly perturbed problems," Applied Mathematics and Computation, vol. 217, no. 8, pp. 36413716, 2010.

[8] M. Kumar, P. Singh, and H. K. Mishra, "A recent survey on computational techniques for solving singularly perturbed boundary value problems," International Journal of Computer Mathematics, vol. 84, no. 10, pp. 1439-1463, 2007.

[9] V. Kumar and B. Srinivasan, "An adaptive mesh strategy for singularly perturbed convection diffusion problems," Applied Mathematical Modelling, vol. 39, no. 7, pp. 2081-2091, 2015.

[10] T. Linß, "Layer-adapted meshes for convection-diffusion problems," Computer Methods in Applied Mechanics and Engineering, vol. 192, no. 9-10, pp. 1061-1105, 2003.

[11] H. G. Roos, M. Stynes, and L. Tobiska, Robust Numerical Methods Singularly Perturbed Differential Equations: Convection-diffusion-reaction and Flow Problems, vol. 24, Springer-Verlag, Berlin, 2008.

[12] V. A. Il'in and E. I. Moiseev, "Nonlocal boundary value problem of the first kind for a Sturm-Liouville operator in its differential and finite difference aspects," Differantial Equations, vol. 23, no. 7, pp. 803-810, 1987.

[13] V. A. Il'in and E. I. Moiseev, "Nonlocal boundary value problem of the second kind for a Sturm-Liouville operator," Differantial Equations, vol. 23, pp. 979-987, 1987.

[14] A. V. Bitsadze and A. A. Samarskii, "On some simpler generalization of linear elliptic boundary value problem," Doklady Akademii Nauk SSSR., vol. 185, pp. 739-740, 1969.

[15] N. Adzic, "Spectral approximation and nonlocal boundary value problems," Novi Sad Journal of Math., vol. 30, no. 3, pp. 1-10, 2000.

[16] D. Herceg and K. Surla, "Solving a nonlocal singularly perturbed nonlocal problem by splines in tension," Univ. $u$ Novom Sadu Zb. Rad. Prirod.-Mat. Fak. Ser. Mathematics, vol. 21, no. 2, pp. 119-132, 1991.

[17] N. Petrovic, "On a uniform numerical method for a nonlocal problem," Univ. u Novom Sadu Zb. Rad. Prirod.-Mat. Fak. Ser. Mathematics, vol. 21, no. 2, pp. 133-140, 1991.

[18] M. Benchohra and S. K. Ntouyas, "Existence of solutions of nonlinear differential equations with nonlocal conditions," Journal of Mathematical Analysis and Applications, vol. 252, no. 1, pp. 477-483, 2000.

[19] T. Jankowski, "Existence of solutions of differential equations with nonlinear multipoint boundary conditions," Computer and Mathematics with Applications, vol. 47, no. 6-7, pp. 1095-1103, 2004.

[20] G. Amiraliyev and M. Çakir, "Numerical solution of the singularly perturbed problem with nonlocal boundary condition," Applied Mathematics and Mechanics, vol. 23, no. 7, pp. 755764, 2002.

[21] M. Cakir, "A numerical study on the difference solution of singularly perturbed semilinear problem with integral boundary condition," Mathematical Modelling and Analysis, vol. 21, no. 5, pp. 644-658, 2016.
[22] M. Cakir and G. M. Amiraliyev, "A finite difference method for the singularly perturbed problem with nonlocal boundary condition," Applied Mathematics and Computation, vol. 160, no. 2, pp. 539-549, 2005.

[23] R. Ciegis, "The numerical solution of singularly perturbed nonlocal problem (in Russian)," Lietuvas Matematica Rink, vol. 28, pp. 144-152, 1988.

[24] R. Ciegis, "The difference scheme for problems with nonlocal conditions," Informatica (Lietuva), vol. 2, pp. 155-170, 1991.

[25] E. Cimen and M. Cakir, "Numerical treatment of nonlocal boundary value problem with layer behaviour," Bulletin of the Belgian Mathematical Society Simon Stevin, vol. 24, no. 3, pp. 339-352, 2017.

[26] Z. Du and L. Kong, "Asymptotic solutions of singularly perturbed second-order differential equations and application to multi-point boundary value problems," Applied Mathematics Letters, vol. 23, no. 9, pp. 980-983, 2010.

[27] M. Kudu and G. M. Amiraliyev, "Finite difference method for a singularly perturbed differential equations with integral boundary condition," International Journal of Mathematics and Computation, vol. 26, no. 3, pp. 72-79, 2015.

[28] M. Sapagovas and R. Ciegis, "Numerical solution of nonlocal problems (in Russian)," Lietuvas Matematica Rink, vol. 27, pp. 348-356, 1987.

[29] M. Cakir, "Uniform second-order difference method for a singularly perturbed three-point boundary value problem," Advances in Difference Equations, vol. 2010, Article ID 102484, 13 pages, 2010.

[30] Z. Cen, "A second-order difference scheme for a parameterized singular perturbation problem," International Journal of Computer Mathematics, vol. 221, no. 1, pp. 174-182, 2008.

[31] Z. Cen, A. Le, and A. Xu, "Parameter-uniform hybrid difference scheme for solutions and derivatives in singularly perturbed initial value problems," Journal of Computational and Applied Mathematics, vol. 320, pp. 176-192, 2017.

[32] C. Clavero, J. L. Gracia, and F. Lisbona, "High order methods on Shishkin meshes for singular perturbation problems of convection-diffusion type," Numerical Algorithms, vol. 22, no. 1, pp. 73-97, 1999.

[33] M. Kumar and S. Chandra Sekhara Rao, "High order parameter-robust numerical method for singularly perturbed reaction-diffusion problems," Applied Mathematics and Computation, vol. 216, no. 4, pp. 1036-1046, 2010.

[34] Q. Zheng, X. Li, and Y. Gao, "Uniformly convergent hybrid schemes for solutions and derivatives in quasilinear singularly perturbed BVPs," Applied Numerical Mathematics, vol. 91, pp. 46-59, 2015.

[35] H. G. Debela and G. F. Duressa, "Uniformly convergent numerical method for singularly perturbedconvection-diffusiontype problems with nonlocal boundary condition," International Journal for Numerical Methods in Fluids, vol. 92, no. 12, pp. 1914-1926, 2020.

[36] M. Cakir, E. Cimen, and G. Amiraliyev, "The difference schemes for solving singularly perturbed three-point boundary value problem," Lithuanian Mathematical Journal, vol. 60, no. 2, pp. 147-160, 2020. 